\title{
Performance Analysis of Diversity Combining over Rayleigh Fading Channels with Impulsive Noise
}

\author{
Zhijiang $X U^{1}$, Yi GONG ${ }^{2}$, Kang $W A N G^{3}$, Yuan $W U^{1}$, Weidang $L U^{1}$, Jingyu HUA ${ }^{1}$ \\ ${ }^{1}$ College of Information Engineering, Zhejiang University of Technology, Liuhe Rd. 288, 310023 Hangzhou, China \\ ${ }^{2}$ Dept. of Electrical and Electronic Engineering, South University of Science and Technology of China, Xueyuan Rd. 1088, \\ 518055 Shenzhen, China \\ ${ }^{3}$ Dept. of Computer and Information Technology, Zhejiang Police College, Binwen Rd. 555, 310053 Hangzhou, China
}

\{zyfxzj, iewuy, luweid, eehjy\}@zjut.edu.cn ${ }^{1}$, gong.y@sustc.edu.cn², wangkang@zjjcxy.cn ${ }^{3}$

Manuscript received February 24, 2016

\begin{abstract}
This paper investigates the performance analysis of diversity combining over flat fading channels subject to additive impulsive noise. Specifically, the impulsive noise follows the symmetric $\alpha$-stable $(S \alpha S)$ distribution. Traditionally, this is a difficult problem since the analytical expression for the probability density function of a general SaS distribution remains unknown. In order to tackle this difficulty, we adopt Fourier power transform to obtain an exact bit error rate (BER) expression for the Genie-aided receiver as well as a BER upper bound for the conventional linear matched filter receiver, without taking any approximation. MonteCarlo simulations are performed to validate our analytical results. The simulation results show that our proposed BER expressions are valid in the whole geometric signal-noiseratio (GSNR) ranges, thus outperforming the previous results valid only at high GSNR values.
\end{abstract}

\section{Keywords}

Alpha Stable Distribution, Diversity Combining, Fourier Power Transform

\section{Introduction}

The additive Gaussian noise model has been widely used in the analysis and design of communication systems since it yields a simple and tractable mathematical model [1]. However, impulsive noises often arise due to automobile spark plugs [2], lighting discharges, underwater sonar, and network interference [3], etc., which demonstrates that the Gaussian noise model may not be accurate in many situations. For example, a power line communication (PLC) channel is mainly affected by the ever-present background noise and the occasional high-amplitude impulses. A standard model for characterizing impulses is to assume Gaussian distributed amplitude and Poisson distributed arrivals [4]. Moreover, studies [5-8] showed that the multiple access interference might result in a Symmetric-Alpha-Stable $(\mathrm{S} \alpha \mathrm{S})$ distribution in a multi-user network with the power-law path loss. The
$\mathrm{S} \alpha \mathrm{S}$ distribution has been used to model the co-channel interference and the aggregate interference in different wireless networks such as cognitive radio networks, UWB networks and cooperative transmission in UWB relays [9]. In fact, the $\alpha$-stable distribution encompasses an important class of distributions which can successfully model a number of impulsive noise processes, because it satisfies the generalized center extreme limit theorem. In particular, the Gaussian distribution can be considered as a special case of the $\mathrm{S} \alpha \mathrm{S}$ distribution [10]. Therefore, we consider the $\mathrm{S} \alpha \mathrm{S}$ distributed impulsive noise in this paper.

In order to overcome the performance degradation of communication systems over a fading channel, the receiver diversity is usually employed. Some results of diversity combining subject to non-Gaussian noise have been obtained in [11], which investigates the performance in the presence of flat fading plus spherically invariant noise. In [12], a statistical model called Middleton's Class-A noise was proposed to model the impulsive noise approximately. The approximation is given by a weighted sum of multiple Gaussian distributions with different variances and amplitudes. In [13], the authors further provided the closed-form bit-error-rate (BER) expressions for the linear diversity combining scheme where the noise follows the Middleton class-A distribution. Furthermore, in [14], [15], the authors investigated the symbol error rate for linear diversity combining schemes in high signal-noise-ratio (SNR) region under the assumption of flat fading plus symmetric non-Gaussian noise. It is noteworthy that all the aforementioned papers assumed the existence of the moments of the noise distribution, which cannot be guaranteed for $\alpha$-stable probability density functions (PDFs).

In [16], the authors analyzed the BER performance for diversity combining over a flat fading channel with the additive noise modeled as $\alpha$-stable distribution. The BER expressions therein are obtained under the high SNR region, in which the PDF of $\alpha$-stable distribution can be approximated by a simple yet accurate function. However, the results in [16] will become inaccurate when the SNR decreases, 
because the approximation used in [16] will become inaccurate. To the best of our knowledge, it is still an open question to explore the analytical error rate performance of diversity combining schemes over flat fading channels, subject to $\alpha$ stable additive noise over entire SNR region of interest. The key difficulty lies in the unknown closed-form expression for the $\mathrm{S} \alpha \mathrm{S}$ distribution at arbitrary SNRs. Therefore, our contributions in this paper are to address this difficulty and to derive the analytical BER performance at arbitrary SNR values. Specifically, to tackle the difficulty, we exploit the special characteristic function (CF) of $\mathrm{S} \alpha \mathrm{S}$ distribution and solve an equivalent problem in the "frequency" domain via the technique of Fourier power transformation [17] for both the Maximal Ratio Combining (MRC) receiver with full sideinformation (which is called as Genie-Aided Receiver in [16], GAR ) and the Linear Matched Filter receiver (LMF) without making any assumption on the SNR region.

The rest of the paper is organized as follows. The $\alpha$ stable distribution and system model are introduced in Sec. 2 . We obtain the exact BER expression for GAR in Sec. 3.1 as well as a BER upper bound for LMF in Sec. 3.2. Monte Carlo simulations are performed in Sec. 4 under high and moderate impulsive noise scenarios. It is shown that the presented analytical results are consistent with the simulation results in all SNR regions. Finally, this paper is concluded in Sec. 5.

Notion: We use boldface type for vectors. $\mathbb{E}[\cdot]$ denotes the statistical expectation; $\|\mathbf{x}\|_{p}$ is the $p$-norm of a vector $\mathbf{x}$; the distribution of a circularly symmetric complex Gaussian random variable with zero mean and variance $\sigma^{2}$ is defined as $C \mathcal{N}\left(0, \sigma^{2}\right)$, and $\sim$ means "distributed as"; $\mathfrak{R}\{\cdot\}, \mathfrak{J}\{\cdot\}$ denote the real and imaginary part of a complex, respectively; $x^{*}$ denotes the conjugate of a complex number $x ; x \triangleq y$ means the expression $y$ is described as $x$ for simplicity.

\section{System Model}

\section{1 $\alpha$-Stable Distribution}

We first briefly review the $\alpha$-stable distribution in this section. A real valued $\alpha$-stable random variable, $w$, has a characteristic function $(\mathrm{CF})$ given by [18]

$$
\phi_{w}(t)=\exp \left(\imath \delta t-|\sigma t|^{\alpha}(1-\imath \beta \operatorname{sign}(t) \zeta(t, \alpha))\right)
$$

where

$$
\zeta(t, \alpha)= \begin{cases}\tan (\pi \alpha / 2) & \alpha \neq 1 \\ \frac{2}{\pi} \log (t) & \alpha=1\end{cases}
$$

In (2), $\alpha \in(0,2], \beta \in[-1,1], \sigma \in(0, \infty)$, and $\delta \in(-\infty, \infty)$ are parameters of the characteristic exponent, skew, scale (or dispersion $\gamma=\sigma^{\alpha}$ ) and shift (or location), respectively. We denote an $\alpha$-stable random variable as $w \sim S_{\alpha}(\sigma, \beta, \delta)$. Random variable $w$ follows symmetric $\alpha$-stable $(\mathrm{S} \alpha \mathrm{S})$ distribution if $\delta=0$ and $\beta=0$. Moreover, if $\sigma=1$, then $w$ is said to be standardized.
For any $\mathrm{S} \alpha \mathrm{S}$ random variable $(\alpha \neq 2)$, only its $p$-order moments exist, where $0 \leq p<\alpha$. Hence, the variance of $\mathrm{S} \alpha \mathrm{S}$ distributed noises, is undefined. As a result, the geometric SNR (GSNR) [19] is used in this paper, i.e.,

$$
\rho=\frac{1}{2 C_{g}}\left(\frac{A_{s}}{S_{0}}\right)^{2}
$$

where $A_{s}, C_{g} \approx 1.78$, and $S_{0}=C_{g}^{\frac{1}{\alpha}-1} \sigma$ denote the amplitude of a modulated signal, the exponential of the Euler constant, and the geometric power of the $\mathrm{S} \alpha \mathrm{S}$ random variable, respectively. The normalization constant " $2 C_{g}$ " is used to ensure that the definition of the GSNR corresponds to that of the standard SNR if the channel noise is Gaussian.

The $\mathrm{S} \alpha \mathrm{S}$ random variable has some useful properties as follows.

Property 1 [20, p20, Proposition 1.3.1] Any real $S \alpha S$ random variable, $w \sim S_{\alpha}(\sigma, 0,0)$ can be written as compound Gaussian, i.e., of the form $w=\sqrt{A} G$, where $A$ and $G$ are independent, with $A \sim S_{\alpha / 2}\left(\left[\cos (\pi \alpha / 4]^{2 / \alpha}, 1,0\right)\right.$ and $G \sim S_{2}(\sigma, 0,0)$ $\left(o r \sim \mathcal{N}\left(0,2 \sigma^{2}\right)\right)$.

Property 2 [20, p10, Property 1.2.1] Let $X_{1}$ and $X_{2}$ be independent random variables with $X_{i} \sim S_{\alpha}\left(\sigma_{i}, \beta_{i}, \mu_{i}\right), i=1,2$. Then $X_{1}+X_{2} \sim S_{\alpha}(\sigma, \beta, \mu)$, with

$$
\sigma=\left(\sigma_{1}^{\alpha}+\sigma_{2}^{\alpha}\right)^{1 / \alpha}, \beta=\frac{\beta_{1} \sigma_{1}^{\alpha}+\beta_{2} \sigma_{2}^{\alpha}}{\sigma_{1}^{\alpha}+\sigma_{2}^{\alpha}}, \mu=\mu_{1}+\mu_{2} \text {. }
$$

Property 3 [20, p16, Property 1.2.15] Let $X \sim S_{\alpha}(\sigma, \beta, \mu)$ with $0<\alpha<2$. Then

$$
\lim _{\lambda \rightarrow \infty} P(X>\lambda)=C_{\alpha}(1+\beta) \sigma^{\alpha} \lambda^{-\alpha}
$$

where $C_{\alpha}=\Gamma(\alpha) \sin (\pi \alpha / 2) / \pi$, and $\Gamma(z)=\int_{0}^{\infty} t^{z-1} e^{-t} \mathrm{~d} t$.

Although a closed-form expression for the PDF, $f_{\alpha}(x) \sim S_{\alpha}(\sigma, \beta, 0)$, does not exist except for a few special cases, asymptotic expansion is well known as $x \rightarrow \infty$, which is given by

$$
f_{\alpha}(x)=(1+\beta) \alpha C_{\alpha} \sigma^{\alpha}|x|^{-\alpha-1}+O\left(|x|^{-2 \alpha-1}\right)
$$

where we write $f(x)=O(g(x))$ as $x \rightarrow a$ to indicate that $\limsup _{x \rightarrow a}|f(x) / g(x)|<\infty$.

Next we define the complex isometric $S \alpha S$ [16] random variables used in the rest of this paper.

Definition 1 If both $G^{R} \triangleq \mathfrak{R}\{G\}, G^{I} \triangleq \mathfrak{J}\{G\}$ are independent identically distributed (i.i.d.) random variables following the zero mean Gaussian distribution with variance $\sigma^{2}$ (i.e., $G^{I}, G^{R} \sim \mathcal{N}\left(0, \sigma^{2}\right)$ ), and $A \sim$ $S_{\alpha / 2}\left(\left[\cos (\pi \alpha / 4]^{2 / \alpha}, 1,0\right)\right.$ is independent of $G^{R}$ and $G^{I}$, then

$$
w=\sqrt{A}\left(G^{R}+{ }_{l} G^{I}\right)
$$

is said to follow the complex isometric $\mathrm{S} \alpha \mathrm{S}$ distribution denoted by $w \sim C S_{\alpha}(\sigma, 0,0)$.

\subsection{System Model}

The considered system model employs one transmitantenna and $L$ receive-antennas. Specifically, the received signal corrupted by the $\mathrm{S} \alpha \mathrm{S}$ impulsive noise can be described by the following equation 


$$
y_{l}=\sqrt{\rho} h_{l} s+w_{l}, \quad l=1, \ldots, L,
$$

where $\rho, h_{l}, s$ and $w_{l}$ represent GSNR, channel fading coefficient, the transmitted signal and the impulsive noise, respectively. Specially, $\rho$ is the GSNR defined by (3). The $l^{\text {th }}$ channel fading coefficient, $h_{l}$, follows $C \mathcal{N}(0,1)$ distribution. The transmitted signal $s \in\{-1,1\}$ is chosen from a binary phase shift keyed (BPSK) constellation. The $l^{\text {th }}$ channel impulsive noise $w_{l}$ follows the i.i.d. $C_{\mathcal{\alpha}}(1,0,0)$ distribution since any scale is subsumed in $\rho$.

By Definition $1, w_{l}$ can be written as

$$
w_{l}=\sqrt{A_{l}}\left(G_{l}^{R}+{ }_{l} G_{l}^{I}\right), \quad l=1, \cdots, L
$$

where $A_{l} \sim S_{\alpha / 2}\left(\left[\cos (\pi \alpha / 4]^{2 / \alpha}, 1,0\right)\right.$ and $\left\{G_{l}^{R}, G_{l}^{I}\right\} \sim$ $\mathcal{N}(0,1)\left(\right.$ or $\left.G_{l} \sim C \mathcal{N}(0,2)\right)$.

\section{Performance Evaluation}

We evaluate the BER performance of different combination schemes in this section. Section 3.1 focuses on the genie-aided receiver and Section 3.2 focuses on the LMF receiver.

\subsection{Genie-Aided Receiver}

Let $\mathbf{h}=\left[h_{1}, \cdots, h_{L}\right], \mathbf{A}=\left[A_{1}, \cdots, A_{L}\right]$ denote the instantaneous channel realizations and a-priori knowledge of the impulsive noises at the receiver, respectively. The socalled Genie-Aided Receiver (GAR) has complete a-priori knowledge of $\mathbf{A}$ and $\mathbf{h}$ [16]. Therefore, the received signal in (8) can be rewritten as

$$
\frac{y_{l}}{\sqrt{A_{l}}}=\sqrt{\frac{\rho}{A_{l}}} h_{l} s+\frac{w_{l}}{\sqrt{A_{l}}}, l=1, \cdots, L .
$$

Since $w_{l} / \sqrt{A_{l}} \sim \mathcal{C N}(0,2)$, the formulation in (8) reduces to diversity combining with Gaussian noise, for which, the optimum combining scheme is maximal ratio combining (MRC), with the $l^{\text {th }}$ combining coefficient equal to $h_{l}^{*} / \sqrt{A_{l}}$. Hence, the decision rule is given by

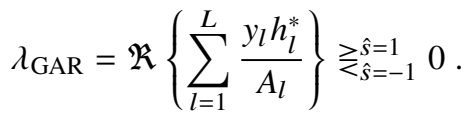

As a result, the corresponding BER conditioned on $\mathbf{A}$ and $\mathbf{h}$ is given by

$$
\operatorname{BER}_{\mathrm{GAR}}(\rho \mid \mathbf{A}, \mathbf{h})=Q\left(\sqrt{\rho \sum_{l=1}^{L} \frac{\left|h_{l}\right|^{2}}{A_{l}}}\right)
$$

where $Q(\cdot)$ is the Gaussian Q-function which can be expressed as [21, pp. 182],

$$
Q(x)=\frac{1}{\pi} \int_{0}^{\pi / 2} \exp \left(-\frac{x^{2}}{2 \sin ^{2} \theta}\right) \mathrm{d} \theta, x>0 .
$$

Since $\left\{h_{l}, l=1,2, \cdots, L\right\}$ are i.i.d. [16] and $h_{l} \sim$ $C \mathcal{N}(0,1),\left|h_{l}\right|^{2}$ follows the exponential distribution with mean 1. The identity $\mathbb{E}_{\mathbf{h}}\left[\exp \left(-\sum_{l=1}^{L} \lambda_{l}\left|h_{l}\right|^{2}\right)\right]=$
$\prod_{l=1}^{L}\left(1+\lambda_{l}\right)^{-1}$ is easily obtained because of the following derivation,

$$
\begin{gathered}
\mathbb{E}_{\mathbf{h}}\left[\exp \left(-\sum_{l=1}^{L} \lambda_{l}\left|h_{l}\right|^{2}\right)\right]=\mathbb{E}_{\mathbf{h}}\left[\prod_{l=1}^{L} e^{-\lambda_{l}\left|h_{l}\right|^{2}}\right] \\
=\prod_{l=1}^{L} \mathbb{E}_{h_{l}}\left[\exp \left(-\lambda_{l}\left|h_{l}\right|^{2}\right)\right]=\prod_{l=1}^{L} \frac{1}{1+\lambda_{l}} .
\end{gathered}
$$

Using this identity and the $Q(\cdot)$ function in (13), we obtain the average BER of GAR with respect to $h$ as

$$
\mathbb{E}_{\mathbf{h}}\left[\operatorname{BER}_{\mathrm{GAR}}(\rho \mid \mathbf{A}, \mathbf{h})\right]=\frac{1}{\pi} \int_{0}^{\pi / 2}\left(\prod_{l=1}^{L} \frac{A_{l}}{A_{l}+\rho_{\theta}}\right) \mathrm{d} \theta
$$

where $\rho_{\theta} \triangleq \frac{\rho}{2 \sin ^{2} \theta}$.

The fact that $\left\{A_{l}, l=1, \cdots, L\right\}$ are i.i.d. [16], the average BER of the GAR with respect to $\mathbf{h}$ and $\mathbf{A}$ is thus given by

$$
\operatorname{BER}_{\mathrm{GAR}}(\rho)=\frac{1}{\pi} \int_{0}^{\pi / 2}\left(\mathbb{E}_{A}\left[\frac{A}{A+\rho_{\theta}}\right]\right)^{L} \mathrm{~d} \theta
$$

where random variable $A$ represents any element of A. Let $f_{\alpha / 2}(A)$ denote the PDF of $A$ with $S_{\alpha / 2}\left([\cos (\pi \alpha / 4)]^{2 / \alpha}, 1,0\right)$ distribution, the expectation on the right-hand side of (16) can be rewritten as

$$
\mathbb{E}_{A}\left[\frac{A}{A+\rho_{\theta}}\right]=\int_{0}^{\infty} \frac{A}{A+\rho_{\theta}} f_{\alpha / 2}(A) \mathrm{d} A .
$$

Unfortunately, the closed-form expression of $f_{\alpha / 2}(A)$ does not exist, except for $\alpha=1$. Eq. (17) cannot be evaluated via direct integration, however, it can be obtained with numerical integral calculation. Under the high GSNR assumption $(\rho \rightarrow \infty)$, the authors of [16] obtained an approximated expression of (17). Substituting the $\operatorname{PDF} f_{\alpha / 2}(A)$ as suggested by (6) into (17), we get

$$
\mathbb{E}_{A}\left[\frac{A}{A+\rho_{\theta}}\right] \approx \Gamma\left(1+\frac{\alpha}{2}\right)\left(\frac{\rho}{2}\right)^{-\frac{\alpha}{2}} \sin ^{\alpha} \theta .
$$

As shown in Fig. 1, the greater GSNR $\rho$ (or $\alpha$ ), the higher the approximation accuracy. In particular, (18) gives a good approximation only in the case of the larger GSNR and moderate impulsive noise. Meanwhile, its approximation accuracy degrades very fast in the case of low GSNR or severe impulsive noise. Therefore, the approximation errors cannot be neglected if the GSNR $\rho$ is medium or small. In order to address this problem, in this paper, we exploit the Fourier power theorem (FPT) [17] to obtain an exact expression of (17). Suppose $f(t)$ and $g(t)$ are two real functions, and the corresponding Fourier transforms are $F(\omega)$ and $G(\omega)$, respectively. According to the FPT, the following formula is workable,

$$
\int_{-\infty}^{\infty} f(t) \cdot g(t) \mathrm{d} t=\frac{1}{2 \pi} \int_{-\infty}^{\infty} F(\omega) \cdot G^{*}(\omega) \mathrm{d} \omega
$$

where $(\cdot)^{*}$ denotes the conjugation of a complex function. We obtain the following result via the FPT.

Theorem 1 The exact BER expression of the Genie Aided Receiver is given by 


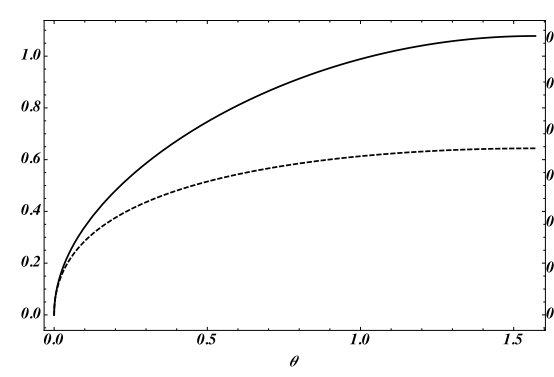

(a) $\alpha=0.5, \rho=0 \mathrm{~dB}$

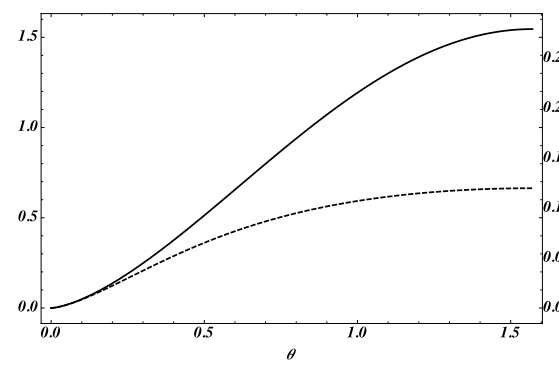

(d) $\alpha=1.5, \rho=0 \mathrm{~dB}$

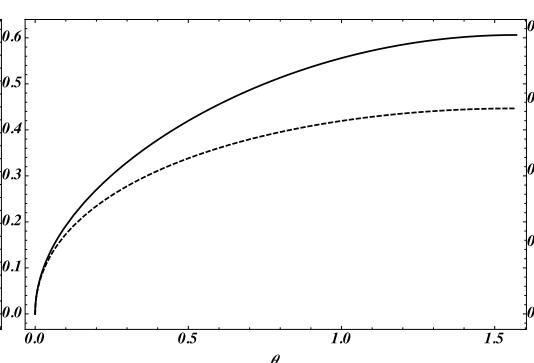

(b) $\alpha=0.5, \rho=10 \mathrm{~dB}$

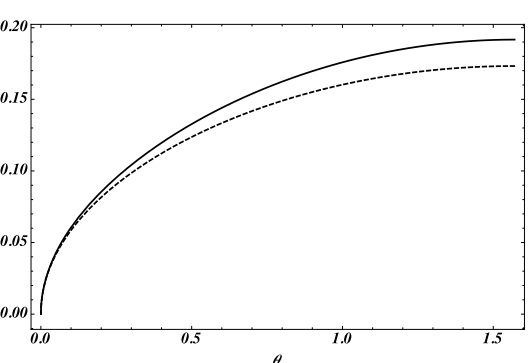

(c) $\alpha=0.5, \rho=30 \mathrm{~dB}$

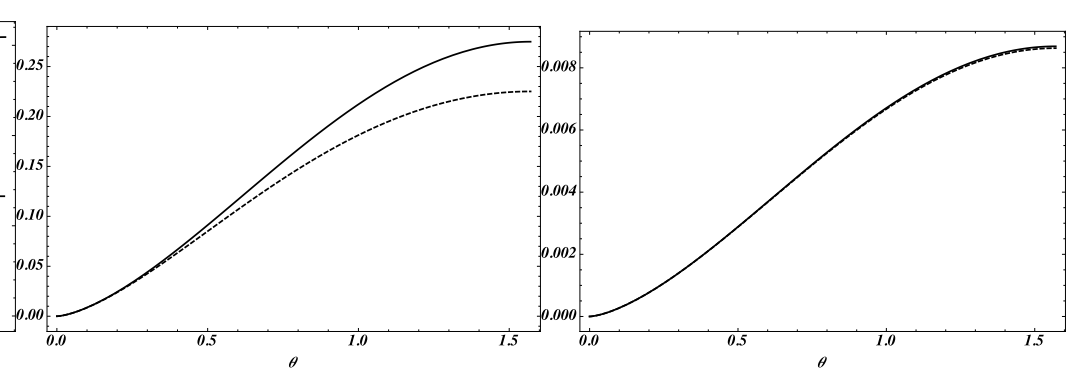

(e) $\alpha=1.5, \rho=10 \mathrm{~dB}$

(f) $\alpha=1.5, \rho=30 \mathrm{~dB}$

Fig. 1. The numerical integral calculation (17) (dotted line) against the approximated version (solid line) (18) with various $\alpha$ and $\rho$ values, where the label of vertical axis is $\mathbb{E}_{A}\left[\frac{A}{A+\rho_{\theta}}\right]$.

$$
\begin{aligned}
& \operatorname{BER}_{\mathrm{GAR}}(\rho)=\frac{1}{\pi} \int_{0}^{\pi / 2}\left(1-\frac{\rho_{\theta}}{\sigma} \times\right. \\
& \left.\int_{0}^{\infty} e^{-\omega^{\alpha / 2}} \sin \left(\frac{\rho_{\theta}}{\sigma} \omega+\tan \left(\frac{\pi \alpha}{4}\right) \omega^{\alpha / 2}\right) \mathrm{d} \omega\right)^{L} \mathrm{~d} \theta
\end{aligned}
$$

where $\sigma \triangleq[\cos (\pi \alpha / 4)]^{2 / \alpha}$, and $\rho$ is GSNR.

Proof: The core of proof is to obtain the closed-form expression of (17). Let $g(A) \triangleq \frac{A \sin ^{2} \theta}{A \sin ^{2} \theta+\rho / 2}=\frac{A}{A+\rho_{\theta}}$, then the expectation on the left-hand side of (17) can be rewritten as $\int_{0}^{\infty} f_{\alpha / 2}(A) \cdot g(A) \mathrm{d} A$.

We cannot solve directly this integral value since there is no closed-form expression for $f_{\alpha / 2}(A)$. As we know, the $\mathrm{PDF}$ and the CF of a stochastic process compose a Fourier transform pair. Fortunately, the Fourier transform of $f_{\alpha / 2}(A)$ (i.e., its CF) and the Fourier transform of $g(A)$ have closedform expressions. Therefore, we solve this problem by using the FPT and obtain the exact expression for (17), which is given as

$\mathbb{E}_{A}\left[\frac{A}{A+\rho_{\theta}}\right]=1-\frac{\rho_{\theta}}{\sigma} \int_{0}^{\infty} e^{-\omega^{\frac{\alpha}{2}}} \sin \left(\frac{\rho_{\theta}}{\sigma} \omega+\tan \left(\frac{\pi \alpha}{4}\right) \omega^{\frac{\alpha}{2}}\right) \mathrm{d} \omega$.

For the details of the proof, please refer to Appendix 6.1.

Remark 1: If GSNR $\rho$ is large enough to make (18) satisfied, then the BER of GAR can be further simplified as

$$
\operatorname{BER}_{\mathrm{GAR}}(\rho) \approx \frac{\Gamma\left(1+\frac{\alpha}{2}\right)^{L} \Gamma\left(\frac{1+L \alpha}{2}\right)}{\sqrt{4 \pi} \Gamma\left(1+\frac{L \alpha}{2}\right)}\left(\frac{\rho}{2}\right)^{-L \alpha / 2}
$$

which is consistent with the previous result in [16, see Eq. (10)].

\subsection{Linear Matched Filtering}

This subsection analyzes the BER performance of linear matched filtering (LMF). The decision rule of LMF is the maximum ratio combining when the noise is Gaussian, which is given by

$$
\lambda_{\mathrm{LMF}}=\sum_{l=1}^{L} \mathfrak{R}\left\{y_{l} h_{l}^{*}\right\} \gtrless_{\hat{s}=-1}^{\hat{s}=1} 0 .
$$

Since the $\mathrm{S} \alpha \mathrm{S}$ noise has infinite variance, the LMF is no longer optimal. Therefore, it is important to quantify its performance under the $\mathrm{S} \alpha \mathrm{S}$ noise.

By definition, BER of LMF is

$$
\operatorname{BER}_{\mathrm{LMF}}(\rho \mid \mathbf{h})=\operatorname{Pr}\left(\lambda_{\mathrm{LMF}}>0 \mid s=-1, \mathbf{h}\right)
$$

where $\lambda_{\mathrm{LMF}}$ is the decision statistic, and $\mathbf{h}=$ $\left[h_{1}, \cdots, h_{L}\right], h_{l} \sim \mathcal{C N}(0,1)$ is the instantaneous channel realizations. Substituting (8), (9) into (23), $\lambda_{\mathrm{LMF}}$ can be written as

$$
\lambda_{\mathrm{LMF}}=s \sqrt{\rho}\|\mathbf{h}\|_{2}^{2}+\sum_{l=1}^{L} \sqrt{A_{l}}\left(G_{l}^{R} h_{l}^{R}+G_{l}^{I} h_{l}^{I}\right)
$$

where $p$-norm $\|\mathbf{h}\|_{p} \triangleq\left(\sum_{l=1}^{L}\left|h_{l}\right|^{p}\right)^{1 / p}, p>0$ and $G_{l}^{R}, G_{l}^{I} \sim$ $\mathcal{N}(0,1)$. Now, conditioned on $\mathbf{h}$ and $\mathbf{A}, G_{l}^{\prime} \triangleq G_{l}^{R} h_{l}^{R}+$ $G_{l}^{I} h_{l}^{I} \sim \mathcal{N}\left(0,\left|h_{l}\right|^{2}\right)$, which together with Property 1, yields $\sqrt{A_{l}} G_{l}^{\prime} \sim S_{\alpha}\left(\left|h_{l}\right| / \sqrt{2}, 0,0\right)$. Using Property 2 to obtain $\sum_{l=1}^{L} \sqrt{A_{l}} G_{l}^{\prime} \sim S_{\alpha}\left(\frac{1}{\sqrt{2}}|| \mathbf{h} \|_{\alpha}, 0,0\right)$, and introducing a standardized $S \alpha S$ random variable $v \sim S_{\alpha}(1,0,0)$, the $\lambda_{\text {LMF }}$ can be represented in terms of $v$. Noting $s=-1$ in (24), the decision statistic, $\lambda_{\mathrm{LMF}}$, is rewritten as 


$$
\begin{gathered}
\lambda_{\mathrm{LMF}}=s \sqrt{\rho}\|\mathbf{h}\|_{2}^{2}+\frac{1}{\sqrt{2}}\|\mathbf{h}\|_{\alpha} v>0 \\
\Rightarrow v>\sqrt{2 \rho}\|\mathbf{h}\|_{2}^{2} /\|\mathbf{h}\|_{\alpha} .
\end{gathered}
$$

Hence, we will derive exact BER expressions for BER upper and lower bounds of LMF in the following theorem.

Theorem 2 The BER upper and lower bounds of LMF are given by

$$
\begin{gathered}
\operatorname{BER}_{\mathrm{LMF}}^{\mathrm{UB}}(\rho)=\frac{1}{2}-\frac{\sqrt{2 \rho} L^{\frac{\alpha-2}{2 \alpha}} \Gamma\left(L+\frac{1}{2}\right)}{\pi \Gamma(L)} \times \\
\int_{0}^{\infty} e^{-\omega^{\alpha}} M\left(\frac{1}{2}+L, \frac{3}{2},-\frac{1}{2} \rho L^{\frac{\alpha-2}{\alpha}} \omega^{2}\right) \mathrm{d} \omega, \\
\operatorname{BER}_{\mathrm{LMF}}^{\mathrm{LB}}(\rho)=\frac{1}{2}-\frac{\sqrt{2 \rho} \Gamma\left(L+\frac{1}{2}\right)}{\pi \Gamma(L)} \times \\
\int_{0}^{\infty} e^{-\omega^{\alpha}} M\left(\frac{1}{2}+L, \frac{3}{2},-\frac{1}{2} \rho \omega^{2}\right) \mathrm{d} \omega
\end{gathered}
$$

where $M(a, b, z)$ is the first kind of Kummer confluent hypergeometric function (KCHF) [23, Chap. 18.6].

Proof: $\lambda_{\mathrm{LMF}}$ is represented in terms of a standardized $\mathrm{S} \alpha \mathrm{S}$ random variable $v \sim S_{\alpha}(1,0,0)$. Using the Hölder inequality and averaging over all channel realizations, we can derive the BER upper-bound as

$$
\operatorname{BER}_{\mathrm{LMF}}^{\mathrm{UB}}(\rho)=\int_{0}^{\infty} f_{\chi_{2 L}}(x) \int_{\sigma_{z} x}^{\infty} f_{\alpha}(v) \mathrm{d} v \mathrm{~d} x
$$

where $f_{\chi_{2 L}}(x)$ represents the PDF of a $\chi$ distribution with $2 L$ degrees of freedom, and $\sigma_{z} \triangleq \sqrt{\rho} L^{\frac{\alpha-2}{2 \alpha}}$.

Since there is no closed-form expression for the PDF $f_{\alpha}(v)$ of $v$, to the best of the authors' knowledge, this integral value is very difficult, if not impossible, to obtain via direct integration. In [24-26], some approximations to the PDF of the $\mathrm{S} \alpha \mathrm{S}$ distribution are proposed. Since the approximation uses simple formula to calculate the PDF, its accuracy can be further improved. Moreover, it is still very complicated to calculate (28) by using approximation method. Again, we turn to solve an equivalent problem in "frequency" domain by using the FPT. Noting that the integral lower limit of $f_{\alpha}(v)$ is $\sigma_{z} x$ instead of 0 , we cannot directly use the FPT. For the details of the proof, please refer to Appendix 6.2.

$\mathrm{KCHF}$ belongs to an important class of special functions in the mathematical physics. By exploiting some properties of KCHF [23, Chap. 18.6], we draw the following remark.

Remark 2: KCHF automatically evaluates to simpler functions for certain parameters, e.g., for $L=1$, $M\left(\frac{1}{2}+L, \frac{3}{2},-z\right)=e^{-z}$. Thus, (27) can be simplified as

$$
\operatorname{BER}_{\mathrm{LMF}}(\rho \mid L=1)=\frac{1}{2}-\sqrt{\frac{\rho}{2 \pi}} \int_{0}^{\infty} e^{-\omega^{\alpha}-\frac{1}{2} \rho \omega^{2}} \mathrm{~d} \omega .
$$

Furthermore, if $\alpha=1$, then (29) can be further simplified as

$$
\operatorname{BER}_{\mathrm{LMF}}(\rho \mid \alpha=1, L=1)=\frac{1}{2}-\frac{1}{2} e^{\frac{1}{2 \rho}} \operatorname{erfc}\left(\sqrt{\frac{1}{2 \rho}}\right)
$$

where the complementary error function $\operatorname{erfc}(x)=$ $\frac{2}{\pi} \int_{x}^{\infty} e^{-t^{2}} \mathrm{~d} t$.
Remark 3: When GSNR $\rho$ is large enough to make $f_{\alpha}(v) \approx \alpha C_{\alpha} v^{-\alpha-1}$ established, upper-bound of LMF can be obtained by direct integral from (28), yielding

$$
\operatorname{BER}_{\mathrm{LMF}}^{\mathrm{UB}}(\rho) \approx \frac{C_{\alpha} \Gamma\left(L-\frac{\alpha}{2}\right)}{\Gamma(L)} L^{1-\alpha / 2}(2 \rho)^{-\alpha / 2}
$$

which is consistent with Theorem 2 in [16].

We will show in Section 4 that the BER upper bound (27b) is in fact very tight for the LMF receiver via numerical simulations.

\section{Simulation Results}

In this section, we verify the results of Theorems 1 and 2 by performing Monte Carlo simulations. To compare with [16], the BERs of GAR and LMF receivers are calculated and compared against the theoretically predicted values/bounds given in Theorems 1 and 2 . The impact of electromagnetic interference from household appliances on digital subscriber loop systems is shown to be well modeled by the $\mathrm{S} \alpha \mathrm{S}$ distribution [27]. Similarly, we also assume that $\alpha=1.43$ which corresponds to the value estimated in [28] for modeling radio frequency interference in laptop receivers. To simulate a "highly impulsive" scenario which corresponds to a path loss exponent of $4(2 / \alpha)$ in an environment where the interfering nodes are scattered according to a spatial Poisson point process on a two dimensional plane [3], $\alpha=0.5$ is also considered. The MATLAB code of the $\mathrm{S} \alpha \mathrm{S}$ distribution is obtained from [29].

Figure 2 shows the performance of GAR over channels with severe (or moderate) impulsive noise where the BER curves based on simulation and theoretical expression (20) are plotted. It is observed that the simulation results match with the theoretical formula (20) in the whole GSNR range. More specifically, the BER performance of GAR gets better when the number of receiver antenna $L$ increases. Especially in the high GSNR, e.g., $\rho>10 \mathrm{~dB}$, the BER drops faster. On the other hand, the performance of GAR with severe impulsive noise is orders of magnitude apart from one with moderate impulsive noise.

Figure 3 shows that the simplified theoretical expression (22) presented in Remark 1 is no longer accurate in the low GSNR range, e.g., $\rho<10 \mathrm{~dB}$ for moderate impulsive noise or even $\rho<30 \mathrm{~dB}$ for severe impulsive noise. It means that it needs higher GSNR to meet the simplified BER formula (22) in severe impulsive noise.

The performance of LMF is examined in Figs. 4-5, where it is demonstrated that the theoretical LMF upperbound is tight in the whole GSNR range for the cases of moderate and severe impulsive noise, respectively. It is observed that the LMF upper-bound is tighter when $L$ is smaller or $\alpha$ is larger. Simulation results show that increasing the number of antennas from one to two bring more performance gain as compared to increasing the antennas number from two to three in the case of moderate impulsive noise (e.g. $\alpha=1.43$ ), 


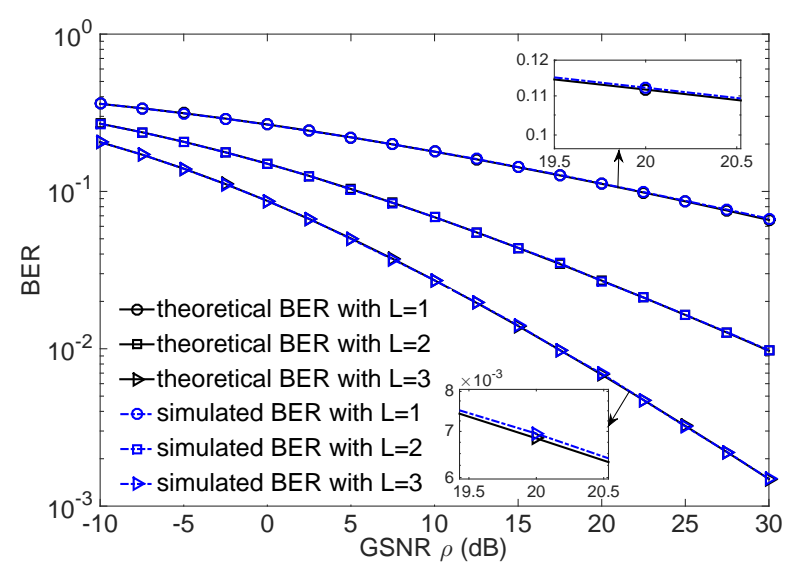

(a) $\alpha=0.50$ (severe impulsive noise)

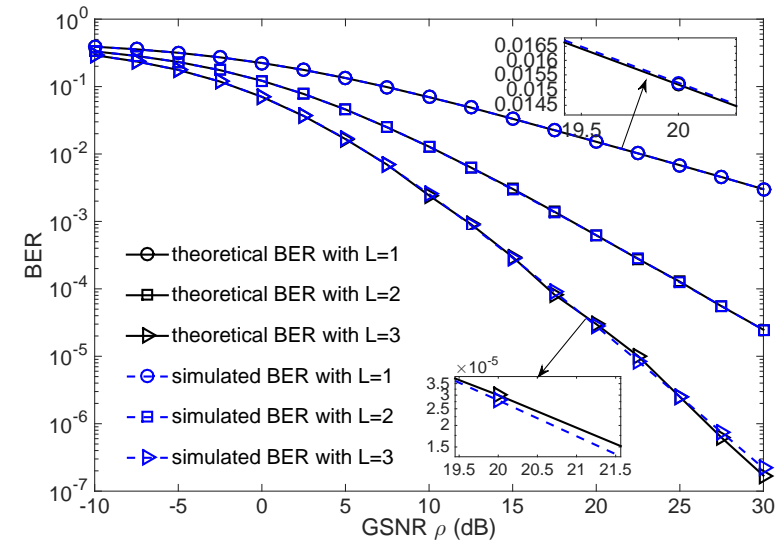

(b) $\alpha=1.43$ (moderate impulsive noise)

Fig. 2. Performance of GAR with $L=1,2,3$.

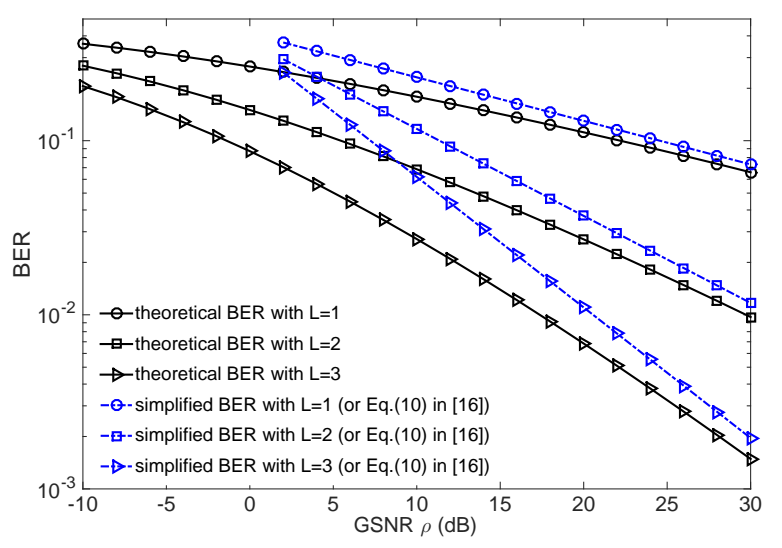

(a) $\alpha=0.50$

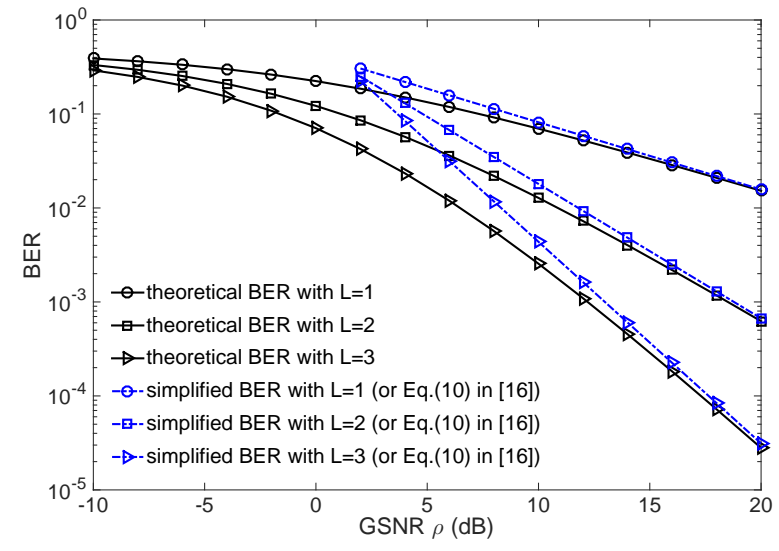

(b) $\alpha=1.43$

Fig. 3. The simplified expression (22) against the proposed expression (20) for GAR.

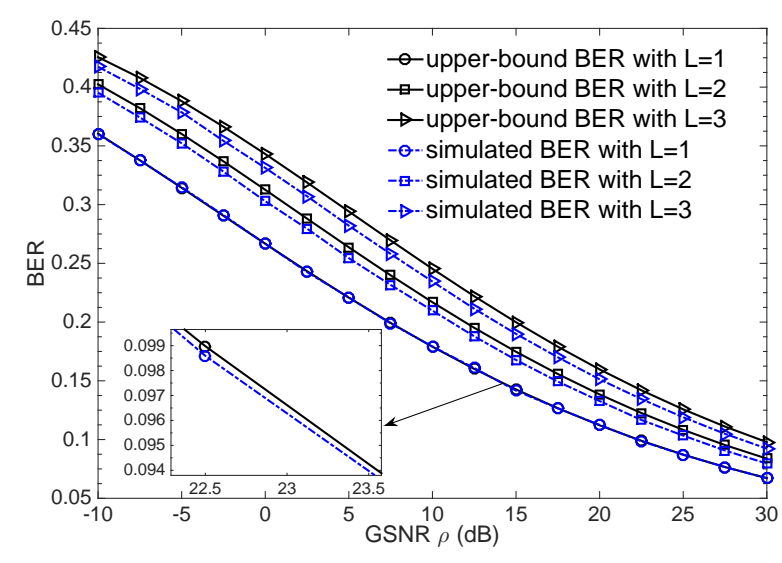

(a) $\alpha=0.50$ (severe implusive noise)

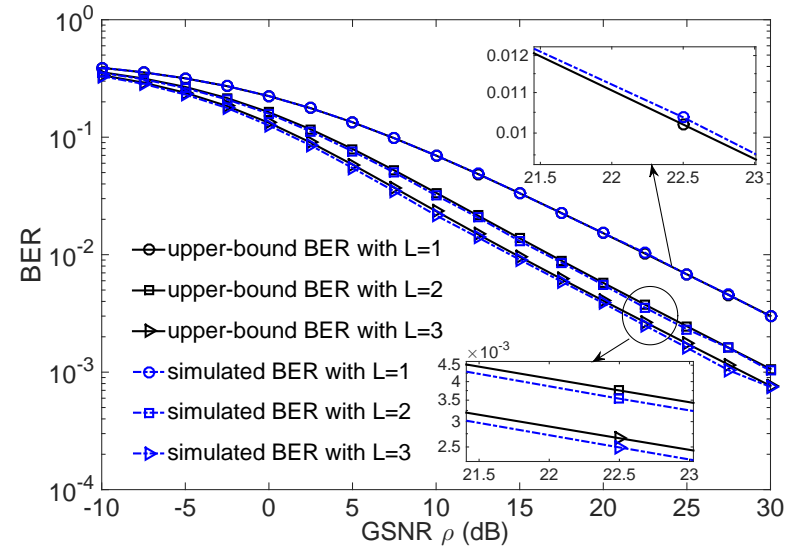

(b) $\alpha=1.43$ (moderate impulsive noise)

Fig. 4. Performance of $\mathrm{LMF}$ with $L=1,2,3$. 


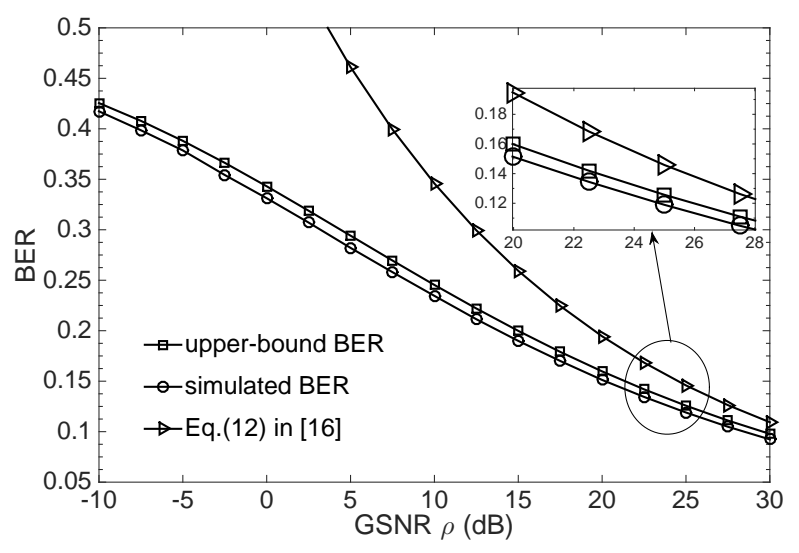

(a) $\alpha=0.50$ (severe implusive noise)

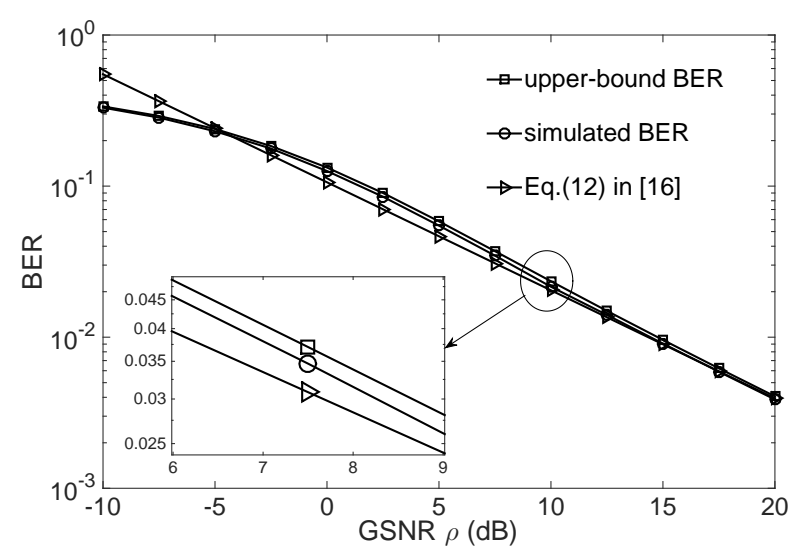

(b) $\alpha=1.43$ (moderate impulsive noise)

Fig. 5. Performance of LMF with the proposed upper-bound expression (27b), where $L=3$.

and the performance gap is limited when further increasing the number of antennas. In the case of severe impulsive noise (e.g. $\alpha=0.5$ ), however, increasing the number of receive antennas is detrimental to the system performance as shown in Fig. 4(a). The behavior of LMF is very different from that of GAR, where employing more antennas always brings higher diversity gain and always improves the system performance.

Figure 5 shows that the theoretical BER upper-bound of LMF in (27b) is tighter than that of [16] in the whole GSNR range. In the case of severe impulsive noise, although GSNR is greater than $20 \mathrm{~dB}$, the theoretically derived LMF bound in [16] differs seriously from the simulation results while our upper-bound is always identical to the simulation results. However, in the case of moderate impulsive noise, when GSNR is greater than $10 \mathrm{~dB}$, they are almost identical.

\section{Conclusions}

In this paper, we analyze the BER performance over fading channels with $\mathrm{S} \alpha \mathrm{S}$ impulsive noise. By exploiting the characteristic function of $\alpha$-stable distribution and solving an equivalent problem in the frequency domain based on the Fourier power transform, we successfully derive the exact BER expressions for both GAR and LMF receivers. Extensive numerical results validate our analytical results, which outperform the existing results that are based on asymptotic approximation.

\section{Appendix}

\subsection{Proof of Theorem 1}

Noting that $f_{\alpha / 2}(A)=0$ when $A<0$, we can extend the range of variable $A$ to satisfy the condition of the Fourier power theorem, i.e.,

$$
\int_{0}^{\infty} f_{\alpha / 2}(A) g(A) \mathrm{d} A=\int_{-\infty}^{\infty} f_{\alpha / 2}(A) g(A) \mathrm{d} A .
$$

The characteristic function of $f_{\alpha / 2}(A)$, denoted by $\phi(\omega ; \alpha / 2,1, \sigma, 0)$, is known as

$$
\phi\left(\omega ; \frac{\alpha}{2}, 1, \sigma, 0\right)=e^{-|\sigma \omega|^{\frac{\alpha}{2}}\left(1-\imath \tan \left(\frac{\pi \alpha}{4}\right) \operatorname{sign}(\omega)\right)} .
$$

And the Fourier transform of $g(A)$ is

$$
\begin{aligned}
\mathbb{F}[g(A)] & =\int_{-\infty}^{\infty} \frac{A}{A+\rho_{\theta}} \exp (\iota \omega A) \mathrm{d} A \\
& =2 \pi \delta(\omega)-\imath \pi \operatorname{sign}(\omega) \rho_{\theta} \exp \left(-\imath \omega \rho_{\theta}\right) .
\end{aligned}
$$

Hence, using the FPT, we obtain

$$
\begin{aligned}
& \int_{-\infty}^{\infty} f_{\alpha / 2}(A) g(A) \mathrm{d} A=\frac{1}{2 \pi} \int_{-\infty}^{\infty} \mathbb{F}\left[f_{\alpha / 2}(A)\right] \mathbb{F}^{*}[g(A)] \mathrm{d} \omega \\
& =\frac{1}{2 \pi} \int_{-\infty}^{\infty} \phi\left(\omega ; \frac{\alpha}{2}, 1, \sigma, 0\right)\left(2 \pi \delta(\omega)-\imath \pi \operatorname{sign}(\omega) \rho_{\theta} e^{-\imath \omega \rho_{\theta}}\right)^{*} \mathrm{~d} \omega \\
& =1+\frac{\imath \rho_{\theta}}{2} \int_{-\infty}^{\infty} e^{-|\sigma \omega|^{\frac{\alpha}{2}}\left(1-\imath \tan \left(\frac{\pi \alpha}{4}\right) \operatorname{sign}(\omega)\right)+\imath \omega \rho_{\theta}} \operatorname{sign}(\omega) \mathrm{d} \omega \\
& =1+\frac{\imath \rho_{\theta}}{2} \int_{-\infty}^{\infty} e^{-|\sigma \omega|^{\frac{\alpha}{2}}} \cos \left(\omega \rho_{\theta}+\tan \left(\frac{\pi \alpha}{4}\right)|\sigma \omega|^{\frac{\alpha}{2}} \operatorname{sign}(\omega)\right) \operatorname{sign}(\omega) \mathrm{d} \omega \\
& -\frac{\rho_{\theta}}{2} \int_{-\infty}^{\infty} e^{-|\sigma \omega|^{\frac{\alpha}{2}}} \sin \left(\omega \rho_{\theta}+\tan \left(\frac{\pi \alpha}{4}\right)|\sigma \omega|^{\frac{\alpha}{2}} \operatorname{sign}(\omega)\right) \operatorname{sign}(\omega) \mathrm{d} \omega .
\end{aligned}
$$

Noting that the integrand of the imaginary term is of odd symmetry, resulting in zero imaginary part. Meanwhile, the integrand of the real term is of even symmetry, thus,

$$
\begin{aligned}
& \int_{-\infty}^{\infty} f_{\alpha / 2}(A) g(A) \mathrm{d} A \\
& =1-\rho_{\theta} \int_{0}^{\infty} e^{-\omega^{\frac{\alpha}{2}} \cos \left(\frac{\pi \alpha}{4}\right)} \sin \left(\rho_{\theta} \omega+\sin \left(\frac{\pi \alpha}{4}\right) \omega^{\frac{\alpha}{2}}\right) \mathrm{d} \omega \\
& =1-\frac{\rho_{\theta}}{\sigma} \int_{0}^{\infty} e^{-\omega^{\frac{\alpha}{2}}} \sin \left(\frac{\rho_{\theta}}{\sigma} \omega+\tan \left(\frac{\pi \alpha}{4}\right) \omega^{\frac{\alpha}{2}}\right) \mathrm{d} \omega .
\end{aligned}
$$

Therefore, substituting (36) into (16), we obtain the BER of the GAR.

\subsection{Proof of Theorem 2}

In this Appendix, we prove Theorem 2. Defining

$$
g(\mathbf{h}) \triangleq\|\mathbf{h}\|_{2}^{2} /\|\mathbf{h}\|_{\alpha}
$$


for brevity and using the Hölder inequality given by

$$
\|\mathbf{h}\|_{2} \leq\|\mathbf{h}\|_{\alpha} \leq L^{\frac{1}{\alpha}-\frac{1}{2}}\|\mathbf{h}\|_{2}
$$

we can derive bound

$$
L^{\frac{1}{2}-\frac{1}{\alpha}}\|\mathbf{h}\|_{2} \leq g(\mathbf{h}) \leq\|\mathbf{h}\|_{2} .
$$

Moreover, defining $x \triangleq \sqrt{2}|| \mathbf{h} \|_{2} \sim \chi_{2 L}$, which represents a $\chi$ distribution (the square root of a $\chi^{2}$ distribution) with $2 L$ degrees of freedom, we obtain $\sqrt{\rho} L^{\frac{1}{2}-\frac{1}{\alpha}} x \leq \sqrt{2 \rho} g(\mathbf{h}) \leq$ $\sqrt{\rho} x$ and thus the following upper and lower bounds conditioned on $\mathbf{h}$,

$$
\begin{aligned}
& \operatorname{Pr}(v>\sqrt{\rho} x) \leq \operatorname{Pr}(v>\sqrt{2 \rho} g(\mathbf{h})) \\
& =\operatorname{BER}_{\mathrm{LMF}}(\rho \mid \mathbf{h}) \leq \operatorname{Pr}\left(v>L^{\frac{\alpha-2}{2 \alpha}} \sqrt{\rho} x\right)
\end{aligned}
$$

where $f_{\chi_{2 L}}(x)=2^{1-L} e^{-x^{2} / 2} x^{2 L-1} \Gamma^{-1}(L)$. Averaging over all channel realizations, with respect to the distribution of $\mathbf{h}$ (or $x$ ), we obtain the BER upper and lower bounds

$$
\begin{array}{r}
\operatorname{BER}_{\mathrm{LMF}}^{\mathrm{UB}}(\rho)=\mathbb{E}_{x}\left[\operatorname{Pr}\left(v>L^{\frac{\alpha-2}{2 \alpha}} \sqrt{\rho} x\right)\right] \\
=\int_{0}^{\infty} f_{\chi 2 L}(x) \int_{L^{\frac{\alpha-2}{2 \alpha}} \sqrt{\rho} x}^{\infty} f_{\alpha}(v) \mathrm{d} v \mathrm{~d} x, \\
\operatorname{BER}_{\mathrm{LMF}}^{\mathrm{LB}}(\rho)=\mathbb{E}_{x}[\operatorname{Pr}(v>\sqrt{\rho} x)] \\
=\int_{0}^{\infty} f_{\chi_{2 L}}(x) \int_{\sqrt{\rho} x}^{\infty} f_{\alpha}(v) \mathrm{d} v \mathrm{~d} x .
\end{array}
$$

However, (41) is hard to solve directly. Therefore, we turn to solve an equivalent problem in "frequency" domain by using the FPT. Denoting $\sigma_{z}^{2} \triangleq \rho L^{\frac{\alpha-2}{\alpha}}$ for brevity, we obtain the BER upper bound as

$$
\begin{aligned}
\operatorname{BER}_{\mathrm{LMF}}^{\mathrm{UB}}(\rho)=\int_{0}^{\infty} f_{\mathcal{X}_{2 L}}(x)\left(\int_{\sigma_{z} x}^{\infty} f_{\alpha}(v) \mathrm{d} v\right) \mathrm{d} x \\
=\int_{0}^{\infty} f_{\alpha}(v)\left(\int_{0}^{\sigma_{z}^{-1} v} f_{\chi_{2} L}(x) \mathrm{d} x\right) \mathrm{d} v \\
=\int_{0}^{\infty} f_{\alpha}(v)\left(1-\Gamma^{-1}(L) \Gamma\left(L, \frac{v^{2}}{2 \sigma_{z}^{2}}\right)\right) \mathrm{d} v \\
=\frac{1}{2}\left(1-\int_{-\infty}^{\infty} f_{\alpha}(v) \Gamma^{-1}(L) \Gamma\left(L, \frac{v^{2}}{2 \sigma_{z}^{2}}\right) \mathrm{d} v\right) \\
=\frac{1}{2}-\frac{\Gamma^{-1}(L)}{4 \pi} \int_{-\infty}^{\infty} e^{-|\omega|^{\alpha}} \times \\
\sqrt{8} \sigma_{z} \Gamma\left(\frac{1}{2}+L\right) M\left(\frac{1}{2}+L, \frac{3}{2},-\frac{1}{2} \sigma_{z}^{2} \omega^{2}\right) \mathrm{d} \omega \\
=\frac{1}{2}-\frac{\sqrt{2} \sigma_{z} \Gamma\left(L+\frac{1}{2}\right)}{\pi \Gamma(L)} \times \\
\int_{0}^{\infty} e^{-\omega^{\alpha}} M\left(\frac{1}{2}+L, \frac{3}{2},-\frac{1}{2} \sigma_{z}^{2} \omega^{2}\right) \mathrm{d} \omega
\end{aligned}
$$

where $\Gamma(a, z)=\int_{z}^{\infty} t^{a-1} e^{-t} \mathrm{~d} t$ presents the incomplete gamma function, and $M(a, b, z)$ denotes the first kind of the Kummer confluent hypergeometric function. $M(a, b, z)$ has the series expansion as $\sum_{k=0}^{\infty} \frac{(a)_{k}}{(b)_{k} k !} z^{k}$, where $(a)_{k}=$ $\prod_{i=0}^{k-1}(a+i)=\Gamma(a+k) / \Gamma(a)$ are Pochhammer symbols with $(a)_{0}=1$ [23].

If GSNR $\rho$ is large enough to make $f_{\alpha}(v) \approx \alpha C_{\alpha} v^{-\alpha-1}$ established, $\operatorname{BER}_{\mathrm{LMF}}^{\mathrm{UB}}(\rho)$ can be obtained by direct integral from (41), yielding

$$
\begin{gathered}
\operatorname{BER}_{\mathrm{LMF}}^{\mathrm{UB}}(\rho) \approx \int_{0}^{\infty} f_{\chi_{2 L}}(x) \int_{\sigma_{z} x}^{\infty} \alpha C_{\alpha} v^{-\alpha-1} \mathrm{~d} v \mathrm{~d} x \\
=\frac{C_{\alpha} \Gamma\left(L-\frac{\alpha}{2}\right)}{\Gamma(L)} L^{1-\alpha / 2}(2 \rho)^{-\alpha / 2}
\end{gathered}
$$

which is consistent with Theorem 2 in [16]. However, when GSNR $\rho$ is small, the upper-bound given by (43) yields large deviation. The reason is explained as follows.

The PDF of the $\mathrm{S} \alpha \mathrm{S}$ distribution, $f_{\alpha}(v)$, does not have a closed-form expression. The requirement of asymptotic expansion (5) in Property 3 is that $v$ is large enough, $v>\theta$ as example (here, $\theta$ is a constant associated with $\alpha$ ). Therefore, the requirement of (43) is $\sigma_{z} x>\theta$. Note that $x$ is a random variable following a $\chi_{2 L}$ distribution. The probability of meeting the requirement (43) is given by

$$
\begin{aligned}
& \operatorname{Pr}\left(x>\sigma_{z}^{-1} \theta\right)=\Gamma\left(L, \frac{\theta^{2}}{2 \sigma_{z}^{2}}\right) \Gamma^{-1}(L) \\
& =\Gamma\left(L, \frac{\theta^{2} L^{\frac{2-\alpha}{\alpha}}}{2 \rho}\right) \Gamma^{-1}(L) .
\end{aligned}
$$

This means that $\sigma_{z} x>\theta$ is satisfied with probability of almost 1 when GSNR $\rho$ is large enough, while for a general $\rho, f_{\alpha}(v) \approx \alpha C_{\alpha} v^{-\alpha-1}$ is not true with a certain probability. Meanwhile, the true value of $f_{\alpha}(v)$ is far less than $\alpha C_{\alpha} v^{-\alpha-1}$ when $v<\theta$, especially at the neighborhood of $v=0$.

\section{Acknowledgments}

The authors would like to thank anonymous reviewers for their time and effort spent in evaluating our manuscript and providing us with constructive comments to improve the quality of the manuscript. This material is based upon work funded by NSFC-Zhejiang Joint Fund for the Integration of Industrialization and Information (No. U1509219), National Natural Science Foundation of China under Grant No. 61471322 \& 61402416, and Zhejiang Provincial Key Laboratory of Communication Networks and Applications.

\section{References}

[1] LEE, J., TEPEDELENLIOĞLU, C. Space-time coding over fading channels with stable noise. In Proceedings International Workshop on Signal Processing Advances in Wireless Communications. Jun. 2011, p. 396-400. DOI: 10.1109/SPAWC.2011.5990438

[2] MIDDLETON, D. Statistical-physical models of electromagnetic interference. IEEE Transactions on Electromagnetic Compatibility, 1977, vol. EMC-19, no. 3, p. 106-127. DOI: 10.1109/TEMC.1977.303527

[3] WIN, M., PINTO, P., SHEPP, L. A mathematical theory of network interference and its applications. In Proceedings of the IEEE. 2009, vol. 97, no. 2, p. 205-230. DOI: 10.1109/JPROC.2008.2008764

[4] ANIRUDDHA, C., ANIRBAN, C., KALYAN, S., et al. Bit error rate of RS coded BFSK in broadband power line channels with background Nakagami and impulsive noise. Physical Communication, 2015, vol. 14, p. 14-23. DOI: 10.1016/j.phycom.2014.11.002 
[5] SOUSA, E. Performance of a spread spectrum packet radio network link in a Poisson field of interferers. IEEE Transactions on Information Theory, 1992, vol. 38, no. 6, p. 1743-1754. DOI: $10.1109 / 18.165447$

[6] YANG, X., PETROPULU, A. Co-channel interference modeling and analysis in a Poisson field of interferers in wireless communications. IEEE Transactions on Signal Processing, 2003, vol. 51, no. 1, p. 64 76. DOI: $10.1109 /$ TSP.2002.806591

[7] HUGHES, B. L. Alpha-stable models of multiuser interference. In Proceedings of IEEE International Symposium on Information Theory. Sorrento (Italy), 2000, p. 383. DOI: 10.1109/ISIT.2000.866681

[8] ILOW, J., HATZINAKOS, D. Analytic alpha-stable noise modeling in a Poisson field of interferers or scatterers. IEEE Transactions on Signal Processing, 1998, vol. 46, no. 6, p. 1601-1611. DOI: $10.1109 / 78.678475$

[9] SALEH, T., MARSLAN, I., EI-TANANY, M. Suboptimal Detectors for Alpha-Stable Noise: Simplifying Design and Improving Performance. IEEE Transactions on Communications, 2012, vol. 60, no. 10, p. 2982-2989. DOI: 10.1109/TCOMM.2012.071812.100789

[10] SHAO, M., NIKIAS, C. Signal processing with fractional low order moments: stable processes and their applications. In Proceedings of IEEE. 1993, vol. 81, no. 7, p. 986-1010. DOI: 10.1109/5.231338

[11] IZZO, L., TANDA, M. Diversity reception of fading signals in spherically invariant noise. In IEEE Proceedings on Communications. 1998, vol. 145, no. 4, p. 272-276. DOI: 10.1049/ip-com:19982131

[12] NAKAGAWA, H., UMEHARA, D., DENNO, S., et al. A decoding for low density parity check codes over impulsive noise channels. In Proceedings International Symposium on Power Line Communnications and its Applications. Vancouver (Canada), 2005, p. 85-89. DOI: 10.1109/ISPLC.2005.1430471

[13] TEPEDELENLIOĞLU, C., GAO, P. On diversity reception over fading channels with impulsive noise. IEEE Transactions on Vehicular Technology, 2005, vol. 54, no. 6, p. 2037-2047. DOI: $10.1109 /$ TVT.2005.853457

[14] NASRI, A., SCHOBER, R., MA, Y. Unified asymptotic analysis of linear modulated signals in fading non-Gaussian noise and interference. In Proceedings IEEE Globecom 2006. San Francisco (USA), 2006, p. 1-6. DOI: 10.1109/GLOCOM.2006.123

[15] NEZAMPOUR, A., NASRI, A., SCHOBER, R., et al. Asymptotic $\mathrm{BEP}$ and SEP of quadratic diversity combining receivers in correlated ricean fading, non-Gaussian noise, and interference. IEEE Transactions on Communications, 2009, vol. 57, no. 4, p. 1039-1049. DOI: 10.1109/TCOMM.2009.04.070085

[16] RAJAN, A., TEPEDELENLIOĞLU, C. Diversity Combining over Rayleigh Fading Channels with Symmetric Alpha-Stable Noise. IEEE Transactions on Wireless Communications, 2010, vol. 9, no. 9, p. 2968-2976. DOI: 10.1109/TWC.2010.071410.100194

[17] BRACEWELL, R. The Fourier Transform and Its Applications. 3rd ed. Singapore: McGraw-Hill, 2000. ISBN: 978-0073039381

[18] NIKIAS, C., SHAO, M. Signal Processing with Alpha-Stable Distributions and Applications. 1st ed. Wiley-Interscience, 1995. ISBN: 978-0471106470

[19] GONZALEZ, J., PAREDES, J., ARCE, G. Zero-order statistics: A mathematical framework for the processing and characteristization of very impulsive signal. IEEE Transactions on Signal Processing, 2006, vol. 54, no. 10, p. 3839-3851. DOI: 10.1109/TSP.2006.880306

[20] SAMORODNITSKY, G., TAQQU, M. Stable Non-Gaussian Random Processes: Stochastic Models with Infinite Variance. New York: Chapman \& Hall, 1994. ISBN: 978-0412051715

[21] GOLDSMITH, G. Wireless Communications. New York: Cambridge University Press, 2005. ISBN: 978-0521837163
[22] UCHAIKIN, V., ZOLOTAREV, V. Chance and Stability, Stable Distributions and Their Applications. Berlin: VSP, 1999. ISBN: 9789067643016

[23] ARFKEN, G. B., WEBER H. J. Mathematical Methods for Physicists. 7th ed. Massachusetts (USA): Academic Press, 2012. ISBN: 9780123846549

[24] XU, J., WANG, K., WU, Y., et al. Minimum-error-based approximation model for symmetric alpha stable distribution. Circuits Systems and Signal Process, 2012, vol. 31, no. 6, p. 2195-2204. DOI: $10.1007 / \mathrm{s} 00034-012-9423-0$

[25] SUREKA, G., KIASALEH, K. Sub-optimum receiver architecture for AWGN channel with symmetric alpha-stable interference. IEEE Transactions on Communications, 2013, vol. 6, no. 5, p. 1926-1935. DOI: 10.1109/TCOMM.2013.022713.120490

[26] CHEN, Y., CHEN, M. Novel S $\alpha$ S PDF approximations and their applications in wireless signal detection. IEEE Transactions on Wireless Communications, 2015, vol. 14, p. 1080-1091. DOI: 10.1109/TWC.2014.2364181

[27] WAN ABDULLAH, W. R., CHUAH, T. C., ZAINAL ABIDIN, A. $\mathrm{N}$., et al. Measurement and verification of the impact of electromagnetic interference from household appliances on digital subscriber loop systems. IET Science, Measurement Technology, 2009, vol. 3, no. 6, p. 384-394. DOI: 10.1049/iet-smt.2009.0002

[28] NASSAR, M., GULATI, K., SUJEETH, A., et al. Mitigating near-field interference in laptop embedded wireless transceivers. In Proceedings IEEE International Conference on Acoustics, Speech and Signal Processing. Nevada (USA), 2008, p. 1405-1408. DOI: $10.1109 /$ ICASSP.2008.4517882

[29] VEILlETTE, M. MATLAB code Alpha-Stable distributions. [online] Cited 2010-06. Available at: math.bu.edu/people/mveillet/html/alphastablepub.html

\section{About the Authors...}

Zhijiang XU was born in 1973. He received his Ph.D. degree in Information and Communication Engineering in 2005, from Zhejiang University, China. He held an appointment as associate professor in the College of Information Engineering at Zhejiang University of Technology, China, from 2007 to now. His research interests include digital communications over fading channels, channel modeling, coding and digital synchronization, etc.

Yi GONG received his $\mathrm{Ph}$.D. degree in electrical engineering from the Hong Kong University of Science and Technology, Hong Kong, in 2002. He then joined the Hong Kong Applied Science and Technology Research Institute, Hong Kong, as a Member of Professional Staff. He is now a Professor at the Southern University of Science and Technology, Shenzhen, China. Prior to his current appointment, he was employed at Nanyang Technological University, Singapore, with which he still has active collaborations. Since 2006, he has served on the editorial board of the IEEE Transactions on Wireless Communications and the IEEE Transactions on Vehicular Technology. His research interests include cognitive radio, full-duplex communications, wireless energy harvesting, and physical layer security for wireless systems. 